M. OKA

KODAI MATH. J.

11 (1988), 441-449

\title{
ON THE STRATIFICATION OF GOOD HYPERSURFACES
}

\author{
By Mutsuo OKA
}

\section{Statement of results.}

Let $f(z)$ be a germ of an analytic function defined in a neighborhood of the origin and let $f(z)=\sum_{\nu} a_{\nu} z^{\nu}$ be the Taylor expansion. We consider the germ of the hypersurface $V=f^{-1}(0)$. We assume that $f$ has a non-degenerate Newton boundary $\Gamma(f)$. The purpose of this paper is to construct a canonical Whitney $b$-regular stratification $\mathcal{S}$ of $V$ which depends only on the Newton boundaries $\{\partial \Gamma(f)\}$. Under the non-degeneracy condition of the Newton boundary, the singular locus of $V$ is the union of several coordinate subspaces $C^{* I}$. However the $b$-regularity for $\left(V^{*}, C^{* I}\right)$ does not hold in general and we have to know the locus where the regularity fails. For this purpose, we introduce the concept of the I-primary boundary components which plays an important role for the stratification of $V$. Its rough description is as follows. Let $P={ }^{t}\left(p_{1}, \cdots, p_{n}\right)$ be a positive rational dual vector and let $I(P)=\left\{1 \leqq i \leqq n ; p_{i}=0\right\}$. The face function $f_{p}(z)$ is defined by the partial sum $\Sigma^{\prime} a_{\nu} z^{\nu}$ for $\nu$ such that $\nu \in \Delta(P)$. Here $\Delta(P)$ is the face of $\Gamma(f)$ where $P$ takes its minimal value $d(P ; f)$. We use the notations of [5]. Assume that $f_{P}(z)=z^{L} g\left(z_{I(P)}\right)$ where $z_{I(P)}$ is the projection of $z$ into the affine coordinate space $C^{I(P)}$. In this case, we say that $f_{P}$ is essentially of $\boldsymbol{z}_{I(P)}$-variables and we denote $g\left(\boldsymbol{z}_{I(P)}\right)$ by $f_{p}^{e}\left(\boldsymbol{z}_{I(P)}\right)$. We consider the variety $V^{*}(P)$ and $\partial V^{*}(P)$ as follows. $V^{*}(P)=\left\{\boldsymbol{z} \in \boldsymbol{C}^{* n} ; f_{P}(\boldsymbol{z})=0\right\}$ and $\partial V^{*}(P)=\left\{\boldsymbol{z}_{I(P)} \in \boldsymbol{C}^{* I(P)} ; f_{p}^{e}\left(\boldsymbol{z}_{I(P)}\right)=0\right\}$. If $f_{P}$ is not essensially of $\boldsymbol{z}_{I(P)}$-variables, $\partial V^{*}(P)$ is $C^{* I(P)}$ by definition. We call $\partial V^{*}(P)$ a I-primary boundary component with respect to $P$ if $V^{*}(P)$ is not empty. Let $V_{p r}$ be the closure of $V^{*}$ in $C^{n}$ and let $V^{* I}=V \cap C^{* I}$ and let $V_{p r}^{* I}=V_{p r} \cap C^{* I}$. Then $V_{p r}^{* I}$ is a union of $I$-primary boundary components (Lemma (3.3)). We say that the hypersurface $V=f^{-1}(0)$ is good if for each subset $I$ of $\{1, \cdots, n\}$ with $|I|>2$, there is at most one $f_{P}$ among $\left\{f_{P} ; I(P)=I\right\}$ such that $f_{P}$ gives a proper $I$-primary boundary component. Here $P$ may not unique. We assume that $V$ is a good hypersurface hereafter. If $V$ has a proper primary boundary component, we denote this component by $\partial V_{p r}^{* I}$. If $V$ does not have proper primary boundary component, $\partial V_{p r}^{* I}=\phi$ by definition. Let $P$ be a positive dual vector and let $I=I(P)$. We say that $V$ satisfies the primary non-degeneracy condition or simply the PND-condition if the following conditions are satisfied for any $P$ such that $V^{*}(P) \neq \phi$. Let $p_{\mathrm{m} \text { n }}$

Received May 30, 1988 
$=$ minimum $\left\{p_{j} ; j \notin I\right\}$.

(PND1) Assume that $f_{P}$ is essentially of $z_{I}$-variables and let $f=f_{P}+\hat{f}$. Write $f_{P}(\boldsymbol{z})=\boldsymbol{z}^{K} f_{P}^{e}\left(\boldsymbol{z}_{I}\right)$ where $K=\left(k_{1}, \cdots, k_{n}\right)$.

(a) (i) $d(P ; f)=0$ or (ii) $d(P ; f)>0$ and $d(P ; \hat{f}) \geqq d(P ; f)+p_{\min }$ or (iii) the variety $\left\{\boldsymbol{z} \in \boldsymbol{C}^{* n} ; f_{P}(\boldsymbol{z})=0, \boldsymbol{z}_{j} \frac{\partial \hat{f}_{P}}{\partial z_{j}}(\boldsymbol{z})-k_{j} \hat{f}_{P}(\boldsymbol{z})=0\right.$ for $\left.\jmath \notin I\right\}$ is empty.

(b) $\partial V^{*}(P)$ is a non-degenerate hypersurface in $C^{* I}$ in an $\varepsilon$-ball $B_{\varepsilon}^{I}$ for some $\varepsilon$.

(PND2) Assume that $f_{P}$ is not essentially of $z_{I}$-variables. For each $z_{I} \in$ $C^{* I} \cap B_{\varepsilon}^{I}$, the fiber $q_{I}^{-1}\left(z_{I}\right)$ is a non-degenerate hypersurface in $C^{I^{c}} \times\left\{z_{I}\right\}$ where $I^{c}$ is the complement of $I$ in $\{1, \cdots, n\}$.

MAIN THEOREM. We assume that $V$ is a good hypersurface which satisfies the PND-condition. Let $\mathcal{S}(I)=\left\{V^{* I}-\partial V_{p r}^{* I}, \partial V_{p r}^{* I}\right\}$ and let $\mathcal{S}=\bigcup_{I} \mathcal{S}(I)$. Then $\mathcal{S}$ is a regular stratification of $V$.

For the stratification of the hypersurfaces which is not good and the stratification of the complete intersection varieties, see [6].

\section{Stratifications.}

Let $V$ be an analytic variety in an open set $D$ of $\boldsymbol{C}^{n}$. We recall the necessary notions of the stratification which is induced by Whitney and Thom. For further details, see $[10,7,3]$. Let $\mathcal{S}$ be a family of subsets of $V$ such that $V$ is covered disjointly by elements of $\mathcal{S}$. $\mathcal{S}$ is called a Whitney stratification if the following conditions are satisfied.

(i) (D-strictness) Each element $M$ of $\mathcal{S}$ (which is called a stratum) is a connected smooth analytic variety such that $\bar{M}$ and $\bar{M}-M$ are closed analytic varieties in $D$. Here $\bar{M}$ is the closure of $M$ in $D$.

(ii) (Frontier property) Let $M$ and $N$ be strata of $\mathcal{S}$ and assume that $M \neq N$ and $M \cap \bar{N} \neq \phi$. Then $M \subset \bar{N}-N$.

We recall the Whitney $b$-condition for a Whitney stratification $\mathcal{S}$. Let $(N, M)$ be a pair of strata of $\mathcal{S}$ with $\bar{N} \supset M$ and let $p$ be a point of $M$. Let $p_{i}$ and $q_{\imath}$ be sequences on $N$ and $M$ respectively. We assume that

$$
p_{\imath} \rightarrow p, \quad q_{\imath} \rightarrow p, \quad T_{p_{i}} N \rightarrow \tau \quad \text { and } \quad\left[p_{i}-q_{\imath}\right] \rightarrow \lambda .
$$

Here the arrows imply the convergence in the respective spaces and $[\boldsymbol{v}]$ is the complex line generated by $\boldsymbol{v}$. Thus $\tau \in G(r, n)(r=\operatorname{dim} N)$ and $\lambda \in G(1, n)=\boldsymbol{P}^{n-1}$ where $G(r, n)$ is the Grassmannian manifold of $r$-planes in $\boldsymbol{C}^{n}$. We say that $(N, M)$ satisfies Whitney $b$-condition at $p$ if $\lambda \in \tau$ for any such sequences. When each pair $(N, M)$ with $M \subset \bar{N}$ satisfies the Whitney $b$-condition at any point $p$ 
of $M$, we call $\mathcal{S}$ a b-regular Whitney stratification. The following proposition is a direct consequence of the Curve Selection Lemma ( 33 of [4] or [1]) and Theorem 17.5 of [10].

Proposition (2.2). Let $p_{i}$ and $q_{i}$ be as in (2.1). Then there are analytic curves $p(t)$ and $q(t)$ defined on the interval $(-\varepsilon, \varepsilon)(\varepsilon>0)$ such that

(i) $p(0)=q(0)=p$ and $p(t) \in N$ for $t \neq 0$ and $q(t) \in M$.

(ii) $T_{p(t)} N \rightarrow \tau$ and $[p(t)-q(t)] \rightarrow \lambda$.

It is known that the $b$-condition for analytic varieties follows from the ratio condition $(R)$ by $[2,9]$. There is also a weaker regularity condition which is called Whitney a-conditıon but this condition results from $b$-condition ([3]).

\section{Non-degenerate hypersurface and primary boundary components.}

Let $f(\boldsymbol{z})=\sum_{\nu} a_{\nu} z^{\nu}$ be an analytic function of $n$ variables which is defined in a neighborhood of the origin. The Newton polyhedron $\Gamma_{+}(f)$ is the convex hull of the union of $\left\{\nu+\boldsymbol{R}_{+}^{n}\right\}$ for $\nu$ such that $a_{\nu} \neq 0$. The Newton boundary $\Gamma(f)$ is the union of the compact faces of the Newton polyhedron. We assume that the Newton boundary $\Gamma(f)$ is non-degenerate. As we are mainly interested in non-isolated singularities, we also use the notation $\partial \Gamma_{+}(f)$ which is the union of the boundaries of $\Gamma_{+}(f)$ which are not necessarily compact. The inclusion $\Gamma(f) \subset \partial \Gamma_{+}(f)$ is obvious by the definition.

Let $\Sigma^{*}$ be a fixed unimodular simplicial subdivision which is compatible with the dual Newton diagrams $\left\{\Gamma^{*}(f)\right\}$ and let $\hat{\pi}: X \rightarrow \boldsymbol{C}^{n}$ be the associated modification map. See [8] and [5] for the definition. Let $V_{p_{r}}$ be the closure of $V^{*}$ and let $\tilde{V}$ be the proper transform of $V_{p r}$ by $\hat{\pi}$. Let $\pi: \tilde{V} \rightarrow V_{p r}$ be the restriction of $\hat{\pi}$ to $\tilde{V}$. For finite vertices $Q_{1}, \cdots, Q_{s}$ of $\Sigma^{*}$, we define a subvariety $E\left(Q_{1}, \cdots, Q_{s}\right)$ of $\tilde{V}$ by $E\left(Q_{1}\right) \cap \cdots \cap E\left(Q_{s}\right)$ and let $E\left(Q_{1}, \cdots, Q_{s}\right)^{*}=$ $E\left(Q_{1}, \cdots, Q_{s}\right)-\bigcup_{P \neq Q_{i}} E(P)$ where $E(P)$ is the divisor of $\tilde{V}$ which corresponds to $P$. Note that $E\left(Q_{1}, \cdots, Q_{s}\right)^{*}$ is non-empty only if $Q_{1}, \cdots, Q_{s}$ are vertices of an $(n-1)$-simplex of $\Sigma^{*}$. The collection of $E\left(Q_{1}, \cdots, Q_{s}\right)^{*}$ gives a regular stratification $\tilde{S}$ of $\tilde{V}$. Let $\sigma=\left(P_{1}, \cdots, P_{n}\right)$. Then we have

$$
\tilde{V} \cap \boldsymbol{C}_{\sigma}^{n}=\left\{\boldsymbol{y}_{\sigma} \in \boldsymbol{C}_{\sigma}^{n} ; f_{\sigma}\left(\boldsymbol{y}_{\sigma}\right)=0\right\}
$$

where $f_{\sigma}\left(\boldsymbol{y}_{\sigma}\right)=f\left(\hat{\pi}\left(\boldsymbol{y}_{\sigma}\right)\right) / \prod_{j=1}^{n} y_{\sigma j}^{d\left(p_{j} ; f\right)}$.

THEOREM (3.2). $\quad \tilde{V}$ is a smooth complex manifold and $\pi: \tilde{V} \rightarrow V_{p r}$ is a proper modification of $V_{p r}$ in the neighborhood of the origin.

The assertion is well known if the origin is an isolated singular point of $V_{p_{r}}$. The general case can be proved similarly. Let $I$ be a subset of $\{1, \cdots, n\}$. We define the coordinate subspace $\boldsymbol{C}^{I}$ and $\boldsymbol{C}^{* I}$ by $\boldsymbol{C}^{I}=\left\{z=\left(z_{1}, \cdots, z_{n}\right) ; z_{j}=0\right.$ if 
$j \notin I\}$ and $\boldsymbol{C}^{* I}=\left\{\boldsymbol{z} \in \boldsymbol{C}^{n} ; z_{3}=0\right.$ iff $\left.j \notin I\right\}$ respectively. For simplicity we usually write $C^{* n}$ instead of $C^{* I}$ if $I=\{1, \cdots, n\}$. We define the I-proper boundary $V_{p r}^{* I}$ of $V$ in $C^{* I}$ by $V_{p r} \cap C^{* I}$. If $I$ is empty, $V_{p r}^{* I}=\{0\}$ by definition. Then we claim:

LEMMA (3.3). The I-proper boundary $V_{p r}^{* I}$ of $V$ is the union of the I-primary boundary components.

Proof. Let $\pi: \tilde{V} \rightarrow V_{p r}$ be the resolution of $V_{p r}$ constructed in $\S 3$. Let $\tilde{V}^{* I}$ be the union of the strata $E\left(P_{1}, \cdots, P_{s}\right)^{*}$ of the stratification $\tilde{S}$ of $\tilde{V}$ such that $\pi\left(E\left(P_{1}, \cdots, P_{s}\right)^{*}\right) \subset C^{* I}$. As $\pi$ is a proper surjective mapping, it is clear that $\pi\left(\tilde{V}^{* I}\right)=V^{* I}$. Let $E\left(P_{1}, \cdots, P_{s}\right)^{*}$ be such a stratum and let $\sigma=\left(P_{1}, \cdots, P_{n}\right)$ be an $(n-1)$-simplex of $\Sigma^{*}$. Let $P=P_{1}+\cdots+P_{s}$. Then $P$ is a positive dual vector with $I(P)=I$. We may assume that $I=\{m+1, \cdots, n\}(m \geqq s)$ for simplicity and $\sigma=\left(p_{\imath j}\right)$ has the following form.

$$
\left(\begin{array}{ll}
A & C \\
0 & B
\end{array}\right)
$$

where $A$ and $B$ are unimodular matrixes of $m \times m$ and $(n-m) \times(n-m)$ respectively. Then Lemma (3.3) follows from the following. $\partial V^{*}(P)$

Sublemma (3.4). The restriction of $\pi$ to $E\left(P_{1}, \cdots, P_{s}\right) *$ is a submersion onto

Proof. Let $\boldsymbol{y}$ be an arbitrary point of $E\left(P_{1}, \cdots, P_{s}\right)^{*}$. Recall that $E\left(P_{1}\right.$, $\left.\cdots, P_{s}\right)^{*}$ is defined by

$$
y_{\sigma 1}=\cdots=y_{\sigma s}=h\left(\boldsymbol{y}_{\sigma}\right)=0
$$

where $h$ is characterized by

$$
h\left(\boldsymbol{y}_{\sigma}\right) \prod_{\imath=1}^{n} y_{\sigma \imath}^{d\left(f ; P_{i}\right)}=f_{P}\left(\hat{\pi}\left(\boldsymbol{y}_{\sigma}\right)\right) .
$$

Note that $\Delta(P)=\int_{\imath=1}^{8} \Delta\left(P_{\imath}\right)$. Thus $h\left(\boldsymbol{y}_{\sigma}\right)$ does not contain the variables $y_{\sigma 1}, \cdots, y_{\sigma s}$. Let $\boldsymbol{z}=\hat{\pi}\left(\boldsymbol{y}_{\sigma}\right)$. Then we have $\boldsymbol{z}_{I}=\left(\boldsymbol{y}_{I}\right)^{B}$ i.e.,

$$
z_{\jmath}=\prod_{\imath=m+1}^{n} y_{\sigma i}^{p_{j i}} \quad(j=m+1, \cdots, n) .
$$

In particular, $\left\{z_{j}\right\}(m+1 \leqq j \leqq n)$ depend only on $y_{\sigma(m+1)}, \ldots, y_{\sigma n}$. Let $E^{*}$ be the subvariety of $\boldsymbol{C}_{\sigma}^{* n}$ defined by $h\left(\boldsymbol{y}_{\sigma}\right)=0 . \quad E^{*}$ is nothing but the product of $\boldsymbol{C}^{* s} \times$ $E\left(P_{1}, \cdots, P_{s}\right)^{*}$. Let $V^{*}(P)$ be the subvariety of the base space $C^{* n}$ which is defined by

$$
V^{*}(P)=\left\{\boldsymbol{z} \in \boldsymbol{C}^{* n} ; f_{P}(\boldsymbol{z})=0\right\} .
$$

It is clear that $\hat{\pi}: E^{*} \rightarrow V^{*}(P)$ is an isomorphism by (3.5). Let $q_{I}: V^{*}(P) \rightarrow \partial V^{*}(P)$ 
and $p: E^{*} \rightarrow E\left(P_{1}, \cdots, P_{s}\right)^{*}$ be the canonical projections. We have the commutative diagram:

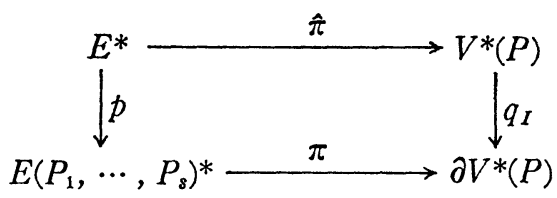

Let $\phi$ be the composition $q \circ \hat{\pi}: E^{*} \rightarrow \partial V^{*}(P)$. By the commutativity of the diagram, $\phi=\pi \circ p$. By the assumption PND1 and PND2, $\phi$ is a submersion. As $\phi=\pi \circ p$, this implies that $\pi: E\left(P_{1}, \cdots, P_{s}\right)^{*} \rightarrow \partial V^{*}(P)$ is a submersion. This completes the proofs of Sublemma (3.4) and Lemma (3.3).

Remark (3.7). Assume that $f\left(\boldsymbol{z}_{I}\right)$ is not identically zero. Then $V^{* I}$ is defined by $f\left(z_{I}\right)=0$. In this case, $f_{P}(z)=f\left(z_{I}\right)$ and for any $P$ with $I(P)=I$. Thus $V^{* I}$ itself is the unique $I$-primary boundary component. In this case, $V$ is nonsingular on $V^{* I}$.

\section{Key Lemma.}

We first consider the following situation. Let $p(t)=\left(p_{1}(t), \cdots, p_{n}(t)\right)$ be an analytic curve defined in the interval $(-1,1)$ with the Taylor expansion $p_{i}(t)=$ $a_{i} t^{b_{i}}+$ (higher terms). We assume that

(i) $f(p(t)) \equiv 0$,

(ii) $a, \neq 0$ for each $j=1, \cdots, n$ and $b_{i}=0$ if and only if $i \in I$.

Let $B={ }^{t}\left(b_{1}, \cdots, b_{n}\right), \boldsymbol{a}=\left(a_{1}, \cdots, a_{n}\right)$. Let $b_{\mathrm{m}_{1 n}}=$ minimum $\left\{b_{j} ; j \notin I\right\}$ and $J_{\min }=\left\{j ; b_{j}=b_{\min }\right\}$. Let $q(t)$ be an analytic curve in $V^{* I}(B)$ with $q(0)=p(0)$. We assume that

(iii) $T_{p(t)} V^{*} \rightarrow \tau$ and $[p(t)-q(t)] \rightarrow \lambda$.

Then we assert

Key Lemma (4.1). $\lambda$ is contained in $\tau$.

Proof. It is well-known that the tangent space $T_{z} V^{*}$ is characterized by $d f(\boldsymbol{z})^{\perp}=\left\{\boldsymbol{v} \in T_{z} \boldsymbol{C}^{n} ; d f(\boldsymbol{z})(\boldsymbol{v})=0\right\}$. Let us consider the limit of $d f(p(t))$. For a real analytic function $k(t)$, we define an integer $\operatorname{ord}(k(t))$ by the order of $k(t)$ at $t=0$. Similarly we define the order of a vector-valued analytic function by the minimum of the order of the coordinate functions. Thus $\operatorname{ord} d(d f(p(t)))$ is the minimum of $\operatorname{ord}\left(\partial f / \partial z_{i}(p(t))\right)$ for $i=1, \cdots, n$. Let $m=\operatorname{ord}(d f(p(t)))$ and let $\vec{\gamma}=$ $d f(p(t)) /\left.t^{m}\right|_{t=0}$. By the PND1-(b)-condition, $m \leqq d(B ; f)$. Let $\vec{\gamma}=\sum_{\imath=1}^{n} \gamma_{i} d z_{\imath}$. Then we have an obvious equality $\tau=\vec{\gamma}^{\perp}$. Considering the leading term of (i), we obtain $f_{B}(\boldsymbol{a})=0$. 
Case (a). Assume that $f_{B}(z)$ is not essentially of $z_{I}$-variables. Then $V^{* I}(B)$ $=C^{* I}$ by the definition. Then by the PND2-condition, there exists an index $j(j \notin I)$ such that $\partial f_{B} / \partial z_{j}(\boldsymbol{a}) \neq 0$ if $\sum_{i \in I}\left|a_{\imath}\right|^{2}$ is small enough. Thus we have $m \leqq d(B ; f)-b_{\min }$. Assume that $m=d(B ; f)-b_{\min }$. Then we must have

$$
\frac{\partial f_{B}}{\partial z_{\jmath}}(\boldsymbol{a})=0 \text { for } j \notin J_{\mathrm{m} 1 \mathrm{n}} \cup I \text { and } \gamma_{\jmath}=\frac{\partial f_{B}}{\partial z_{\jmath}}(\boldsymbol{a}) \text { for } j \in J_{\mathrm{m} 1 \mathrm{n}} \text {. }
$$

If $m<d(B ; f)-b_{\mathrm{min}}$, we have that

$$
\gamma_{3}=0 \quad \text { for } \quad j \in J_{\mathrm{m}_{1 n}} \cup I .
$$

Note that $\gamma_{2}=0$ for $i \in I$ in both cases. This implies that $\vec{\gamma} \mid \boldsymbol{C}^{I}=0$.

Now we consider the line $[p(t)-q(t)]$. Let $k=\operatorname{ord}(p(t)-q(t))$. As $q(t) \in C^{* I}$, it is easy to see that $1 \leqq k \leqq b_{\min }$. Let $\vec{\lambda}=(p(t)-q(t)) /\left.t^{k}\right|_{t=0}$. By the definition of $\lambda$, we have that $[\vec{\lambda}]=\lambda$. If $k<b_{\mathrm{m}_{1 n}}, \vec{\lambda}$ is a vector in $C^{I}$. In this case, it is clear that $\vec{\gamma}(\vec{\lambda})=0$. Assume that $k=b_{\min }$. Then $\lambda_{j}=a$, if $j \in J_{\min }$ and $\lambda_{j}=0$ if $j \notin J_{\min } \cup I$. We consider the equality

$$
\begin{aligned}
0 & \equiv \sum_{j=1}^{n} \frac{\partial f}{\partial z_{\jmath}}(p(t)) \frac{d p_{j}(t)}{d t} \\
& \equiv\left[\sum_{j \in I} \frac{\partial f_{B}}{\partial z_{\jmath}}(\boldsymbol{a}) b_{j} a_{\jmath}\right] t^{d(B ; f)-1}+(\text { higher terms). }
\end{aligned}
$$

Thus we obtain the equality

$$
\sum_{\jmath \notin I} \frac{\partial f_{B}}{\partial z_{\jmath}}(\boldsymbol{\alpha}) b_{j} a_{\jmath}=0 .
$$

If $m<d(B ; f)-b_{\mathrm{m}_{1 n}}, \vec{\gamma}(\vec{\lambda})=0$ is immediate from (4.3). Assume that $m=d(B ; f)$ $-b_{\mathrm{min}}$. By (4.2) and (4.4), we can see easily that $\vec{\gamma}(\vec{\lambda})=0$. Here $\vec{\lambda}$ is identified with the tangent vector $\sum_{j=1}^{n} \lambda_{j} \frac{\partial}{\partial z_{j}}$ at $p(0)$.

Case (b). Assume that $f_{B}(\boldsymbol{z})$ is essentially of $\boldsymbol{z}_{I}$-variables. Let $f_{B}(\boldsymbol{z})=\boldsymbol{z}^{L} f_{B}^{e}(\boldsymbol{z})$ where $z^{L}$ is a monomial in the variables $\left\{z_{j} ; j \notin I\right\}$. Then $V^{* I}(B)=\left\{f_{B}^{e}\left(z_{I}\right)=0\right\}$ and $\operatorname{ord}\left(f_{B}(p(t))\right)=\operatorname{ord}\left(p(t)^{L}\right)=d(B ; f)$. We have two equalities :

$$
\sum_{\jmath=1}^{n} \frac{\partial f}{\partial z_{\jmath}}(p(t)) \frac{d p_{\jmath}(t)}{d t} \equiv 0 \text { and } \sum_{i \in I} \frac{\partial f_{B}^{e}}{\partial z_{\imath}}(q(t)) \frac{d q_{i}(t)}{d t} \equiv 0 .
$$

Let $\beta=\operatorname{ord}\left(f_{B}^{e}(p(t))\right)$ and $\delta=\operatorname{ord}(\hat{f}(p(t)))$. First we assume that PND1-(a)-(ii) holds. As $f(p(t))=f_{B}(p(t))+\hat{f}(p(t)) \equiv 0$, we have

$$
\beta+d(B ; f)=\delta \geqq d(B ; \hat{f})
$$

where $\hat{f}_{B}(\boldsymbol{z})$ is the secondary face function of $f$ with respect to the weight $B$. The equality holds if and only if $\hat{f}_{B}(\boldsymbol{a}) \neq 0$. We consider the equality which follows immediately from (4.5). 


$$
\begin{aligned}
& \sum_{\jmath=1}^{n} \frac{\partial f}{\partial z_{\jmath}}(p(t)) \frac{d}{d t}\left[p_{j}(t)-q_{j}(t)\right]+ \\
& \sum_{i \in I}\left[\frac{\partial f}{\partial z_{\imath}}(p(t))-\frac{\partial f_{B}}{\partial z_{\imath}}(p(t))\right] \frac{d q_{i}(t)}{d t}+ \\
& \sum_{i \in I} p(t)^{L}\left[\frac{\partial f_{B}^{e}}{\partial z_{\imath}}(p(t))-\frac{\partial f_{B}^{e}}{\partial z_{\imath}}(q(t))\right] \frac{d q_{i}(t)}{d t} \equiv 0 .
\end{aligned}
$$

By the assumption, $p_{j}(t) \equiv q_{j}(t)$ modulo $\left(t^{k}\right)$ for any $j$. This implies that $\operatorname{ord}\left[\frac{\partial f_{B}^{e}}{\partial z_{\imath}}(p(t))-\frac{\partial f_{B}^{e}}{\partial z_{\imath}}(q(t))\right] \geqq k$. Thus the order of the last sum is at least $d(B ; f)+k$. On the other hand, we have

$$
\operatorname{ord}\left(\frac{\partial f}{\partial z_{\imath}}(p(t))-\frac{\partial f_{B}}{\partial z_{\imath}}(p(t))\right) \geqq d(B ; \hat{f}) \geqq d(B ; f)+b_{\min } \quad(i \in I)
$$

by PND1-(a)-(ii) where $\hat{f}=f-f_{B}$. As $k \leqq b_{\min }$, the order of the second sum in (4.7) is also at least $d(B ; f)+k$. The order of the first sum in (4.7) is (at least) $m+k-1$. As $m \leqq d(B ; f)$ by the PND1-(b)-condition and $k \leqq b_{\min }$, the coefficient of $t^{m+k-1}$ of (4.7) is equal to $\vec{\gamma}(\vec{\lambda})$. Thus we conclude that $\vec{\gamma}(\vec{\lambda})=0$. Assume (a)-(i) $: d(B ; f)=0$. We consider the following equality instead of (4.7).

$$
\begin{aligned}
& \sum_{j=1}^{n} \frac{\partial f}{\partial z_{\jmath}}(p(t)) \frac{d}{d t}\left[p_{j}(t)-q_{\jmath}(t)\right]+ \\
& \sum_{i \in I}\left[\frac{\partial f}{\partial z_{\imath}}(p(t))-\frac{\partial f}{\partial z_{\imath}}(q(t))\right] \frac{d q_{i}(t)}{d t} \equiv 0 .
\end{aligned}
$$

Here we have used the equality $\frac{\partial f}{\partial z_{\imath}}(q(t))=\frac{\partial f_{B}}{\partial z_{\imath}}(q(t))$. By the PND1-(b)-condition, $m=0$. Thus by a similar argument, we have $\vec{\gamma}(\vec{\lambda})=0$. Note that $m=$ $d(B ; f)$ if the PND1-(a)-condition is satisfied.

Assume that PND1-(a)-(iii) holds. We may assume that $d(B ; \hat{f})<d(B ; f)$ $+b_{\text {min }}$. We consider $(4.7)$ again. The order of the last sum is at least $d(B ; f)$ $+k$. We can write $f_{B}^{e}(p(t))=\lambda t^{\theta}+($ higher terms) by $(4.6)$ where $\theta=d(B ; \hat{f})-$ $d(B ; f)$. Note that $\theta \leqq \beta$. As $f(p(t)) \equiv 0$, we have that $\hat{f}_{B}(\boldsymbol{a})+\lambda \boldsymbol{a}^{K}=0$. Thus we have

$$
\frac{\partial f}{\partial z_{\jmath}}(p(t))=\eta_{j} t^{d(B ; \hat{f})-b_{\jmath}}+(\text { higher terms }) \quad \text { for } j \notin I
$$

where $\eta_{\jmath}=\frac{\partial \hat{f}_{B}}{\partial z_{\jmath}}(\boldsymbol{a})+\lambda k_{j} \boldsymbol{a}^{K} / a_{\jmath}=\left(a_{j} \frac{\partial \hat{f}_{B}}{\partial z_{\jmath}}(\boldsymbol{a})-k_{j} \hat{f}_{B}(\boldsymbol{a})\right) / a_{\jmath}$. As $f_{B}(\boldsymbol{a})=0$, there exists an index $j_{0} \notin I$ such that $\eta_{\rho_{0}} \neq 0$ by the PND1-(a)-(iii) condition. Thus the order of the first term of $(4.7)$ is at most $d(B ; \hat{f})-b_{\jmath_{0}}+k-1$. The order of the second term is at least $d(B ; \hat{f})$. As $k<b_{\min }$, we have the inequality: $d(B ; \hat{f})$ $-b_{\jmath_{o}}+k-1<d(B ; \hat{f})$. By the assumption that $d(B ; \hat{f})<d(B ; f)+b_{\min }$, we have 
also the inequality: $d(B ; \hat{f})-b_{\jmath_{0}}+k-1<d(B ; f)+k$. Therefore we conclude as before that $\vec{\gamma}(\vec{\lambda})=0$. This completes the proof of Lemma (4.1).

\section{Proof of Main Theorem.}

In this section, we will prove Main Theorem in $\S 1$. Let $Y$ and $Z$ be a pair of strata of $\mathcal{S}$ such that $\bar{Y} \cap Z \neq \phi$. We assume that $Y \in \mathcal{S}(J)$ and $Z \in \mathcal{S}(K)$. Then we must have $J \supset K$. If $J=K$, the $b$-regularity is obvious as $V$ is good. Thus we may assume that $J \neq K$. If $Y$ is an open dense stratum in $C^{* J}$, the $b$-regularity for $(Y, Z)$ is again obvious. Thus we assume that $\bar{Y} \neq \boldsymbol{C}^{J}$. Let $p(t)$ and $q(t)$ be real analytic curves defined on $(-1,1)$ such that (i) $p(0)=q(0)$ $\in Z$. (ii) $p(t) \in Y$ for $t>0$. (iii) $q(t) \in Z$ for $t \geqq 0$. Assume that the tangent space $T_{p(t)} Y$ converges to $\tau$ and the line $[p(t)-q(t)]$ converges to $\lambda . \quad Y$ is a non-degenerate hypersurface defined by $f_{P}^{e}\left(\boldsymbol{z}_{J}\right)=0$ for some $P$ with $I(P)=J$. Assume that $p_{j}(t)=a_{j} t^{b}+$ (higher terms) for $j \in J$. For brevity's sake, we assume that $J=\{1, \cdots, m\}$. Let $B={ }^{t}\left(b_{1}, \cdots, b_{m}\right)$ and $\boldsymbol{\alpha}=\left(a_{1}, \cdots, a_{m}\right)$. As $p(0)=q(0)=\boldsymbol{a}_{\boldsymbol{I}}$ $\in Z, K=I(P)$. By looking at the leading terms of the equality $h(p(t)) \equiv 0$, we can see that $\boldsymbol{a}_{K}$ belongs to the $K$-primary component $Y^{* K}(B)$. Let $R=P+r Q$ for a sufficiently small $r>0$. Then it is an easy linear algebra to see the following.

(i) $\left(f_{P}\right)_{B}=f_{R}$. (ii) The secondary face function $\hat{f}_{R}$ of $f$ with respect to $R$ is equal to the secondary face function of $f_{P}$ with respect to $B$.

Thus the PND-condition for $f$ implies the PND-condition for $f_{P}$. Now we use Lemma (4.1) to obtain the regularity for the pair $(Y, Z)$. This completes the proof of Main Theorem.

Example (5.1). Let $f(z)=\left(z_{1} z_{2}\right)^{2}\left(z_{3}^{5}+z_{4}^{5}\right)+\left(z_{3} z_{4}\right)^{2}\left(z_{1}^{5}+z_{2}^{5}\right)$. Then the singular locus of $V$ is the union of the two dimensional coordinate planes $C^{I}$ for $|I|=2$. Let $I=\{1,2\}$. Then by an easy calculation, we have a proper primary boundary components defined by $C: z_{1}^{5}+z_{2}^{5}=0$. $C$ consists of five lines, say $C_{1}, \cdots, C_{5}$. Thus $\mathcal{S}(I)=\left\{C^{* I}-C, C_{1}, \cdots, C_{5}\right\}$. The same is true for $I=\{3,4\}$. Thus the stratification of $V$ consists of the following strata: $V^{*}, C^{* I}(I \neq\{1,2\},\{3,4\})$, $C^{* 11,2\}}-C, C^{*\{3,4\}}-D, C_{\imath}, D_{\imath}(i=1, \cdots, 5), C^{\{j\}}(j=1, \cdots, 4),\{0\}$ where $D=\bigcup_{\imath=1}^{5} D_{i}$ $=\left\{z_{3}^{5}+z_{4}^{5}=0\right\}$.

\section{REFERENCES}

[1] H. HАмм, Lokale topologische Eigenschaften komplexer Räume, Math. Ann., 191 (1971), 235-252.

[2] J.C. Kuo, The ratio test for analytic Whitney stratifications, in Proceedings of Liverpool singularities symposium, Springer Lecture Note, 192 (1971), 141-149.

[3] J. Mather, Stratifications and Mappings, in Dynamical Systems, ed. Peixoto (1973), 195-232. 
[4] J. Milnor, Singular Points of Complex Hypersurface, Annals Math. Studies, 61, Princeton Univ. Press, Princeton, 1968.

[5] M. OKA, On the Resolution of Hypersurface Singularities, Advanced Study in Pure Mathematics, 8 (1986), 405-436.

[6] M. OKA, Canonical stratification of complete intersection varieties, preprint, 1988.

[7] R. Tном, Ensembles et morphismes stratifiés, Bull. Amer. Math. Soc., 75 (1969), 240-284.

[8] A.N. VARChENKo, Zeta-Function of Monodromy and Newton's Diagram, Inventiones Math., 37 (1976), 253-262.

[9] J.P. VERDIER, Stratifications de Whitney et théorème de Bertini-Sard, Inventiones Math, 36 (1976), 295-312.

[10] H. Whitney, Tangents to analytic variety, Ann. Math., 81 (1964), 496-546.

DePARTMENT OF MATHEMATICS

TOKYO INSTITUTE OF TECHNOLOGY 
